\title{
Metastasis of Sigmoid Colon Cancer to the Breast and Uterus: A Case-Study
}

\author{
Andee Dzulkarnaen Zakaria ${ }^{1}$, Amer Hayat Khan ${ }^{2 *}$, Muhammad Abdul Hadi ${ }^{3}$ and Baharudin lbrahim $^{2}$
}

${ }^{1}$ Department of Surgery, School of Medical Sciences, Universiti Sains Malaysia, Health Campus, Kelantan, Malaysia

${ }^{2}$ Department of Clinical Pharmacy, School of Pharmaceutical Sciences Universiti Sains Malaysia, 11800 Penang, Malaysia

${ }^{3}$ School of Healthcare, University of Leeds, LS2 9UT Leeds, United Kingdom

\begin{abstract}
Introduction: Colon cancer is the second most common cancer after breast and the most common cancer among males in Peninsular Malaysia. Metastasis of colon cancer to the uterus and breast rarely occur, but still the incidence could not be ignored as it could possibly happen to anyone with the disease.

Clinical Presentation and Intervention: A 32-years old Malay woman complaining of pain in the right hypochondria region radiating to the back and right shoulder. The pain was constant, with moderate intensity and relieved temporarily by analgesics (Paracetamol). She also noticed a lump in her left breast following Breast Self Examination (BSE). Patient was admitted to Hospital Universitiy Sains Malaysia (HUSM) with no signs of shortness of breath, chest pain or hemoptysis. The patient had history of sigmoid colon cancer (Duke's B) and had undergone sigmoid colectomy two years ago and completed 25 cycles of local radiotherapy and concurrent 12 cycles of systemic chemotherapy. The ultrasound of pelvic region revealed metastasis to pelvic organ.

Conclusion: Surgery is the most common treatment for colorectal cancer and may be curative for localized cancers. Radiotherapy and chemotherapy be used as an adjunct treatment after surgery to increase disease free survival and overall survival but recurrence may still occur.
\end{abstract}

\section{Introduction}

Colorectal cancer occurrence and mortality rates vary around the world. Globally, colorectal cancer is the third most commonly diagnosed cancer in males and second in females. In 2008, 1.2 million new cases and more than 600,000 deaths were estimated globally [1].

Colon cancer is in fact the most common gastrointestinal cancer in Malaysia. A total of 2,866 cases were registered with National Cancer Registry of Malaysia in 2006 representing only 13.2\% of all registered cases. The overall Age Standardized Incidence (ASR) was 18.4 per 100,000 populations. The incidence was higher among males (ASR 21.6 per 100,000) compared to females (ASR 15.4 per 100,000 population). Furthermore, the incidence was higher among Chinese (21.4 per 100,000 populations) as compare to Indian and Malay races [2].

In the early stages, people with colon cancer often have no symptoms at all. Typical signs and symptoms of colon cancer include a change in the bowel habits, including diarrhea or constipation or a change in the consistency of stool for more than a couple of weeks, rectal bleeding, persistent abdominal discomfort, such as cramps, gas or pain, weakness or fatigue and an unexplained weight loss.

The cancer typically starts in the lining of the bowel and if left untreated, it can grow into the muscle layers underneath, and metastases to many other parts of body through the bowel walls.

Metastases to the breast from extramammary malignancies are rare and account for only $0.43 \%$ of all breast malignancies. Lymphoma, melanoma, sarcoma, lung carcinoma and ovarian tumor are common extramammary primary malignancies that metastasize to the breast. Cases of metastasis from primary colorectal neoplasm to the breast are extremely rare, and very limited literature is available. Furthermore, metastases to the uterus from extraintestinal cancers are significantly rarer than metastases to the ovaries.

\section{Case Presentation}

A 32-years old Malay woman complaining of pain in right hypochondrial region radiating to the back and right shoulder for 3 months duration. The pain was constant, with moderate intensity and relieved temporarily by analgesics (Tablet Paracetamol). There were no signs of shortness of breath, chest pain or either hemoptysis.

Patient had first presented to the Hospital Universiti Sains Malaysia (HUSM) two years ago, complaining of lower abdominal pain (more on the left side/left iliac fossa) for two months. The pain was colicky in nature, non-radiating, and associated with constipation. She also had history of passing out mucus mixed with blood with stools. Colonoscopy was done which revealed features of colitis and present of constricting tumour at sigmoid colon $25 \mathrm{~cm}$ from anal verge. Computered Tomography (CT) scan showed the features of proximal sigmoid colon carcinoma with partial bowel obstruction and no distant metastasis. Histopathology report confirmed moderately differentiated adenocarcinoma.

Patient underwent sigmoid colectomy with colorectal anastomosis but complicated with anastomosis leak. Post-operation, she was subjected to laparotomy and peritoneal lavage. Patients completed 25 cycles of local radiotherapy and 12 cycles of concurrent systemic chemotherapy. After three months, colonoscopy was performed again, and revealed mild radiation proctitis, with normal proximal stump, as shown in figure 1.

*Corresponding author: Amer Hayat Khan, Department of Clinical Pharmacy, School of Pharmaceutical Sciences, Universiti Sains Malaysia, 11800 Penang, Malaysia, Tel: 006-014-9227580; Fax: 00604657 0017; E-mail: amerhayat@ymail.com

Received January 08, 2013; Accepted April 09, 2013; Published April 13, 2013

Citation: Zakaria AD, Khan AH, Hadi MA, Ibrahim B (2013) Metastasis of Sigmoid Colon Cancer to the Breast and Uterus: A Case-Study. Trop Med Surg 1: 118.

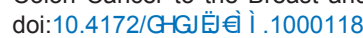

Copyright: (c) 2013 Zakaria AD, et al. This is an open-access article distributed under the terms of the Creative Commons Attribution License, which permits unrestricted use, distribution, and reproduction in any medium, provided the original author and source are credited. 


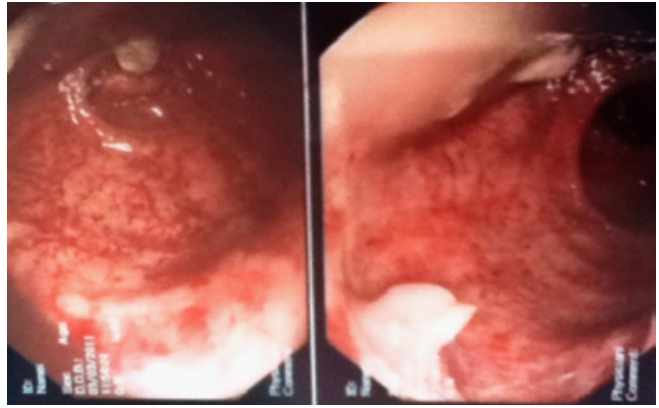

Figure 1: Mild Proctitis, with normal proximal stump.

With the interval of additional three months, patient underwent reversal of sigmoid colostomy with mucus fistula. The Ultrasonograph (USG) revealed no liver metastasis. Her carcinoembryonic (CEA) levels were in normal range. The surveillance colonoscopy was done within further 3 months, which was normal. Meanwhile the pelvic USG was done which revealed adnexal mass possible of pedunculated uterine fibroid and hepatomegaly.

During her regular follow-up in April 2012 at Oncology Clinic, she complains of a lump in her left breast in left outer quadrant following BSE. The lump was about $3 \mathrm{~cm}$ in diameter, non-tender, hard, mobile and gradually increased in size. She had no family history of breast cancer [3].

\section{Discussion}

Colon carcinoma is usually a slowly growing tumor which begins to spread locally around the intestinal wall and to some extent longitudinally [4]. This local spread frequently cause intestinal obstruction before it penetrates adjacent structures. However, penetration of the intestinal serous coat is appropriate to occur and, according to the segment involved, contiguous structures may become involved due to direct spread of the tumor. Distant metastases of colon carcinoma may be passed by the lymphatic stream to the regional lymph nodes draining the colon and by the blood-stream to the liver. Nevertheless, involvement of the female genital tract is a uncommon event and most commonly affects ovaries [5].

Sigmoid colectomy is an excision of a portion or a whole part of sigmoid colon with oncological resection. The safety of colorectal surgery, as performed in patients with colorectal cancer and inflammatory bowel disease, has improved dramatically over the last 50 years due to improvements in preoperative preparation, antibiotic prophylaxis, surgical technique, and postoperative management [5-8].

Radiation therapy for colorectal cancer lowers the risk of cancer recurrence by about $7 \%$. Without radiation, the disease recurs in $50 \%$ of patients with rectal cancer. In the present case, despite undergoing 25 cycle of radiotherapy, the cancer had even spread to uterus and left breast.

Pain in the right hypochondriac region is usually produced by diseases of the liver and gallbladder, hepatic flexure of the colon, diseases of the right chest or right hemi-diaphragm, and disorders of the nervous system, muscle, and bone. The most important diseases include acute and chronic cholecystitis, biliary colic, cancer of the liver or gallbladder, abscess of the liver or pancreas, acute or chronic hepatitis, right hemi-diaphragmatic pleurisy, subphrenic abscess, duodenal ulcer, right segmental or intercostal neuralgia, postcholecystectomy syndrome, right-sided slipped costal cartilage, pneumonia, and pleurisy [6]. In this patient, her pain at the right hypochondriac region could possibly be one of the conditions stated above, or perhaps the factors are still unknown.

Regarding pain management, Tramadol was used to relieve moderate to acute pain. Tramadol relieves pain by inhibiting the ascending pain pathway in the central nervous system. Since this patient was having an acute pain during patient admission, the use of Tramadol was appropriate. Nevertheless, it is not recommended to be used for a prolonged period of time, potentially as it can induce dependence and should be tempered down to avoid withdrawal symptoms [4]. Paracetamol can be used to control prolonged pain as it is one of the safest analgesic.

\section{Conclusion}

Proper and close surveillance can prevent recurrence colorectal cancer through the early detection after removal of primary cancerous lesions and post operative adjunct chemoradiotherapy. Most of the guidelines of colorectal cancer recommend surveillance tools such as clinical assessment, serial colonoscopy, CT scan and CEA level as standard to detect recurrence colorectal cancer especially in first 2 years. Chemoradiotherapy can be used as an adjunct treatment post operatively to increase disease free survival and overall survival, but sometimes recurrence locally or extraintestinal recurrence as shown in this case can still occur despite undergoing surgery and chemoradiotherapy.

\section{References}

1. Jemal A, Bray F, Center MM, Ferlay J, Ward E, et al. (2011) Global cancer statistics. CA Cancer J Clin 61: 69-90.

2. Dahlui M, Ramli S, Bulgiba AM (2011) Breast cancer prevention and control programs in Malaysia. Asian Pac J Cancer Prev 12: 1631-1634.

3. Narringahon C, Natindim ME, Nueva LJ, Omul D, Ordanza A, et al. (2011) A Case Study on Sigmoid Colon Cancer Stage 3 (T3N0).

4. Walfisch S, Lapid O, Yanai-Inbar I, Piura B (1999) Sigmoid colon carcinoma metastatic to the myometrium. Eur J Obstet Gynecol Reprod Biol 86: 65-68.

5. Mazur MT, Hsueh S, Gersell DJ (1984) Metastases to the female genital tract Analysis of 325 cases. Cancer 53: 1978-1984.

6. Georgiannos SN, Chin J, Goode AW, Sheaff M (2001) Secondary neoplasms of the breast: a survey of the 20th Century. Cancer 92: 2259-2266.

7. Noh KT, Oh B, Sung SH, Lee RA, Chung SS, et al. (2011) Metastasis to the breast from colonic adenocarcinoma. J Korean Surg Soc 81 Suppl 1: S43-S46.

8. Nakagami K, Takahashi T, Sugitani K, Sasaki T, Ohwada S, et al. (1999) Uterine cervix metastasis from rectal carcinoma: a case report and a review of the literature. Jpn J Clin Oncol 29: 640-642. 